\title{
Semiclassical zero-temperature corrections to Schwarzschild spacetime and holography
}

\author{
A. Fabbri ${ }^{a}{ }^{*}$ S. Farese ${ }^{a},^{\dagger}$ J. Navarro-Salas ${ }^{a},{ }^{\ddagger}$ G. J. Olmo ${ }^{b}, \S$ and H. \\ Sanchis-Alepuz ${ }^{a}$, \\ a) Departamento de Física Teórica and IFIC, Centro Mixto Universidad de Valencia- \\ CSIC. Facultad de Física, Universidad de Valencia, Burjassot-46100, Valencia, Spain. \\ b) Department of Physics, University of Wisconsin-Milwaukee, P.O. Box 413, Milwaukee, \\ Wisconsin, 53201 USA
}

\begin{abstract}
Motivated by the quest for black holes in $A d S$ braneworlds, and in particular by the holographic conjecture relating $5 D$ classical bulk solutions with $4 D$ quantum corrected ones, we numerically solve the semiclassical Einstein equations (backreaction equations) with matter fields in the (zero temperature) Boulware vacuum state. In the absence of an exact analytical expression for $\left\langle T_{\mu \nu}\right\rangle$ in four dimensions we work within the s-wave approximation. Our results show that the quantum corrected solution is very similar to Schwarzschild till very close to the horizon, but then a bouncing surface for the radial function appears which prevents the formation of an event horizon. We also analyze the behavior of the geometry beyond the bounce, where a curvature singularity arises. In the dual theory, this indicates that the corresponding $5 D$ static classical braneworld solution is not a black hole but rather a naked singularity.
\end{abstract}

PACS numbers: 04.62.+v, 04.70.Dy, 11.25.Tq

\footnotetext{
*afabbri@ific.uv.es

$\dagger$ farese@ific.uv.es

†jnavarro@ific.uv.es

\$olmoalba@uwm.edu

『helios.sanchis@ific.uv.es
} 


\section{Introduction}

The study of quantum effects in black hole spacetimes comes back to the early seventies, when Hawking discovered [1] that black holes evaporate by emission of thermal radiation (see also 2, 3]). This result generated enormous interest in the subject, especially after Hawking himself speculated [4] that the evaporation process will lead to the disappearance of the black hole and the information about its formation will be lost forever. This is a radical conclusion, as it implies that in the quantum theory the whole process of black hole formation and evaporation is nonunitary.

It is clear, however, that the approximation considered to derive this result, i.e. the quantization of matter fields in the fixed classical background describing the formation of a Schwarzschild black hole, and even the framework used, the semiclassical theory of gravity (see, for instance, [5, 6, 17]), cannot lead to a reliable resolution of this paradox. At a certain point during the evolution the quantum effects will backreact and modify significantly the background geometry, which therefore cannot be considered as fixed, nor evolved in a quasi-static approximation. Moreover, once the black hole has reached the Planck size quantum gravitational effects will become important and cannot be neglected anymore." Thus it is no wonder that still today Hawking's provocation continues to raise much debate and although most of the people do not want to give up unitarity [8] (and between them, remarkably, now Hawking himself [9]), a definitive answer on whether and how information is recovered in black hole evaporation is still lacking.

To take into account self-consistently the backreaction effects one needs to solve exactly the semiclassical Einstein equations

$$
G_{\mu \nu}\left(g_{\alpha \beta}\right)=\frac{8 \pi G}{c^{4}}\left\langle\Psi\left|T_{\mu \nu}\left(g_{\alpha \beta}\right)\right| \Psi\right\rangle
$$

for the metric $g_{\alpha \beta}$, where the quantity on the right hand side represents the expectation value of the stress-energy tensor operator of the matter fields in a suitable quantum state $|\Psi\rangle$.

In the fixed Schwarzschild background

$$
d s^{2}=g_{\mu \nu}^{s c h w} d x^{\mu} d x^{\nu}=-\left(1-r_{S} / r\right) c^{2} d t^{2}+\frac{d r^{2}}{\left(1-r_{S} / r\right)}+r^{2} d \Omega^{2},
$$

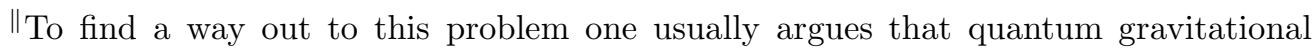
effects should always be negligible compared to those due to a large number $N$ of matter fields.
} 
where $r_{S}=2 G M / c^{2}$ is the Schwarzschild radius, three inequivalent quantum vacuum states can be defined. The first is the Boulware state $|B\rangle[10$, probably the most natural one, constructed by requiring that in the asymptotic region, where the metric becomes minkowskian, it reduces to Minkowski ground state $|M\rangle$. It has the property that $\left\langle B\left|T_{\mu \nu}\right| B\right\rangle$ vanishes asymptotically, but the drawback is a strong divergence at the horizon $r=r_{S}$ [11. One can circumvent this difficulty by introducing a new quantum vacuum state $|H\rangle$, called the Hartle-Hawking state [12, such that $\left\langle H\left|T_{\mu \nu}\right| H\right\rangle$ is regular at the horizon. However one pays a price, i.e. this stress tensor is nonvanishing at large $r$ and describes thermal radiation at the Hawking temperature

$$
T=\frac{\hbar c^{3}}{G k_{B}} \frac{1}{8 \pi M}
$$

where $k_{B}$ is Boltzman's constant. The associated physical situation is that of a black hole in a cavity, in thermal equilibrium with its own radiation. The third possibility, the Unruh state $|U\rangle[13$, is constructed in such a way as to reproduce the late time behaviour of the quantum matter fields in the classical background of a collapsing star forming a black hole. By requiring that no particles are present at infinity in the past and that $\left\langle U\left|T_{\mu \nu}\right| U\right\rangle$ is regular at the future horizon one finds, as a consequence, an outgoing flux of thermal radiation (the Hawking flux) in the asymptotic future.

By inserting the fixed background expression $\left\langle\Psi\left|T_{\mu \nu}\left(g_{\alpha \beta}^{s c h w}\right)\right| \Psi\right\rangle$ in the right hand side of Eqs. (11) one can solve perturbatively the backreaction equations at $O(\hbar)$ to find the first order quantum corrections $\delta g_{\alpha \beta}$ to the Schwarzschild metric, i.e. $g_{\alpha \beta}=g_{\alpha \beta}^{s c h w}+\delta g_{\alpha \beta}$. This is a good approximation to the full solution of (11) only when $\delta g_{\alpha \beta} \ll g_{\alpha \beta}^{s c h w}$, i.e. when the quantum terms are small compared to the background.** In the case of large mass being the Hawking temperature very small this condition is satisfied, for an evaporating black hole, for most of its lifetime, but eventually at the late stages of the evaporation one faces the problems mentioned above. Turning to the static configurations, this is a good approximation in the Hartle-Hawking case since $\left\langle H\left|T_{\mu \nu}\right| H\right\rangle$ never gets large, while for Boulware it is certainly valid at infinity but not at the horizon due to the divergence of the quantum stress tensor there. The problem of understanding how, in this case, the quantum effects

** The natural coordinates to be used in this context are the Schwarzschild ones given in (2). In more general terms, this approximation scheme is valid whenever the quantum terms generate a correction to the curvature which is small compared to its background value. 
modify the structure of the classical horizon is not an easy one. One usually disregards this question since Boulware state describes the vacuum polarization around a static star whose radius is bigger than $r_{S}$, and therefore this divergence is not physically relevant.

The motivation for our work comes from braneworld physics, where much work is being done on the search for black hole solutions in the RandallSundrum model RS2 [14]. This is technically a very involved situation and nobody so far has achieved the goal of finding a five-dimensional solution describing a black hole localized on the brane [16. An interesting physical interpretation of this fact comes from the application of the holographic AdS/CFT correspondence [15], for which classical 5D bulk solutions are dual to 4D self-consistent semiclassical configurations where gravity is coupled to quantum matter fields [17. If this is so then a classical static 5D "black hole" will be mapped to a 4D static solution of the backreaction equations. Staticity naturally selects the Hartle-Hawking and Boulware states. If, in addition, we require that the $4 \mathrm{D}$ configuration be asymptotically flat then the choice must be the Boulware state. That this is the correct choice is supported by the fact that for large radius the corrections to the Newtonian potential in this state match those calculated classically in 5D [18].

In order to solve the backreaction equations in the Boulware vacuum the exact expression of $\left\langle B\left|T_{\mu \nu}\left(g_{\alpha \beta}\right)\right| B\right\rangle$ for an arbitrary geometry is needed. No such expression exists in four dimensions (an analytic approximation for static spherically symmetric spacetimes has however been developed in [19]). The situation greatly improves if one restricts to the s-wave approximation for spherically symmetric backgrounds. Within this context, in section 2 we review the classical and semiclassical theory of gravity coupled to a massless and minimally coupled scalar field. Since in this case the expression of $\left\langle B\left|T_{\mu \nu}\left(g_{\alpha \beta}\right)\right| B\right\rangle$ is available, we end the section by writing down the relevant backreaction equations. These equations cannot be exactly solved analytically, and we approach the problem in two steps. First, in section 3, we consider the equations arising in the Polyakov theory, which can be derived from ours using a near-horizon approximation for the scalar field (this amounts to neglecting backscattering effects in the propagation of the matter fields). These equations are decoupled, in the sense that one can derive an equation relating the conformal factor of the metric $\rho$ $\left(d s^{2}=e^{2 \rho}\left(-c^{2} d t^{2}+d x^{2}\right)+r^{2} d \Omega^{2}\right)$ as a function of the radius $r$ only (or $\phi$ or $z$ through the definitions $r=r_{0} e^{-\phi}=r_{0} z$ according to the convenience) which is then integrated numerically and from which one can derive the de- 
pendence on the spatial coordinate $\rho=\rho(x)$ and $r=r(x)$. The quantum corrected solution is very similar to the Schwarzschild solution from infinity till very close to the classical horizon $r=r_{S}$, where, as expected, big differences emerge. In particular, there exists a timelike surface $r=r_{B}$ where the radial function $r$ bounces (i.e. the two-spheres reach a minimum radius $r_{B}$ and then they start to increase) and, beyond it, a null curvature singularity with infinite radius at a finite affine distance. Armed with these techniques and results we face, in section 4 , the full backreaction equations in the s-wave approximation, which are much more complicated. The differences with respect to the previous case are that the bounce is located closer to the classical horizon $r=r_{S}$ and that the curvature singularity is now timelike and has finite radius. Finally, in section 5 we summarize our conclusions.

\section{Gravity coupled to a massless scalar field in the $s$-wave approximation}

It is convenient, in the context of the $s$-wave approximation, to work with spherically reduced theories. Under the spherically symmetric ansatz

$$
d s_{(4)}^{2}=d s_{(2)}^{2}+r^{2} d \Omega^{2}
$$

the Hilbert-Einstein action

$$
S_{g}^{(4)}=\frac{c^{3}}{16 \pi G} \int d^{4} x \sqrt{-g^{(4)}} R^{(4)}
$$

reduces to

$$
S_{g}=\frac{c^{3}}{4 G} \int d^{2} x \sqrt{-g}\left[r^{2} R+2\left(1+|\nabla r|^{2}\right)\right],
$$

where the geometrical quantities refer to the radial part $d s_{(2)}^{2}$ of the fourdimensional metric. Note that the radial variable $r$ here plays the role of a scalar field with a non-trivial coupling to the radial sector of the metric. Einstein's equations for spherically symmetric configurations in vacuum can be rewritten as

$$
\begin{aligned}
& \frac{2}{\sqrt{-g}} \frac{\delta S_{g}}{\delta g^{a b}} \equiv \frac{c^{3}}{4 G}\left[-2 r \nabla_{a} \nabla_{b} r+g_{a b}\left(2 r \square r-1+|\nabla r|^{2}\right)\right]=0, \\
& \frac{2}{\sqrt{-g}} \frac{\delta S_{g}}{\delta r} \equiv \frac{c^{3}}{G}[r R-2 \square r]=0 .
\end{aligned}
$$


The solution of these equations is the Schwarzschild geometry

$$
\begin{aligned}
d s_{(2)}^{2} & =-\left(1-\frac{2 G M}{c^{2} r}\right)\left(d t^{2}-d r^{* 2}\right) \\
r^{*} & =r+\frac{2 G M}{c^{2}} \ln \left(1-\frac{2 G M}{c^{2} r}\right),
\end{aligned}
$$

where $r^{*}$ is the so-called "tortoise" coordinate.

Turning to the matter sector, let us consider the action for a minimally coupled massless scalar field (in Gaussian units)

$$
S_{m}^{(4)}=-\frac{1}{8 \pi} \int d^{4} x \sqrt{-g^{(4)}}(\nabla f)^{2}
$$

In the background $d s_{(4)}^{2}=g_{a b} d x^{a} d x^{b}+r^{2} d \Omega^{2}$ the field $f$ can be expanded in spherical harmonics, of which we pick up only the $s$-wave component

$$
f=f\left(x^{a}\right) \equiv \frac{f_{l=0}}{r} Y_{00}
$$

Under this assumption, integration of the angular variables in (10) leads to

$$
S_{m}=-\frac{1}{2} \int d^{2} x \sqrt{-g} r^{2}(\nabla f)^{2} .
$$

Varying this action with respect to the radial part of the metric we obtain a two-dimensional stress-energy tensor

$$
-\frac{2 c}{\sqrt{-g}} \frac{\delta S_{m}}{\delta g^{a b}} \equiv T_{a b},
$$

which is related to the radial components of the corresponding four-dimensional one by the relation

$$
T_{a b}^{(4)}=\frac{T_{a b}}{4 \pi r^{2}}
$$

Moreover, by varying (12) with respect to $r$ we get the expression for the angular components of the four-dimensional stress-energy tensor

$$
T_{\theta \theta}^{(4)}=\frac{T_{\varphi \varphi}^{(4)}}{\sin ^{2} \theta}=-\frac{r c}{8 \pi \sqrt{-g^{(2)}}} \frac{\delta S_{m}}{\delta r} .
$$




\subsection{Semiclassical theory}

The advantage of the approximation considered is that in this case, unlike the full four-dimensional treatment, one can provide an analytic expression for the expectation values of all components of the stress-energy tensor. We shall briefly review the main steps involved. The details can be found in [7]. To this end it is very convenient to parameterize the radial part of the four dimensional metric in conformal gauge as

$$
d s_{(2)}^{2}=-e^{2 \rho} d x^{+} d x^{-},
$$

and moreover it is also useful to parameterize the radial coordinate as follows

$$
r=r_{0} e^{-\phi}
$$

One can univocally provide an expression for $\left\langle T_{ \pm \pm}^{(4)}\right\rangle$ and $\left\langle T_{\theta \theta}^{(4)}\right\rangle$ by imposing two simple conditions:

- the covariant conservation laws

$$
\nabla^{\mu}\left\langle T_{\mu \nu}^{(4)}\right\rangle=0
$$

which can be rewritten as

$$
\nabla^{a}\left\langle T_{a b}\right\rangle=\nabla_{b} \phi \frac{1}{\sqrt{-g}}\left\langle\frac{\delta S_{m}}{\delta \phi}\right\rangle
$$

- at an arbitrary point $X$ of the spacetime manifold the expectation values of the quantum stress-energy tensor $\left\langle T_{ \pm \pm}\left(x^{ \pm}(X)\right)\right\rangle$ reduce to the normal ordering ones $\left\langle: T_{ \pm \pm}\left(x^{ \pm}(X)\right):\right\rangle$ when using a locally inertial frame $\xi_{X}^{\alpha}$ based on that point

$$
\left\langle T_{ \pm \pm}\left(\xi_{X}^{\alpha}(X)\right)\right\rangle=\left\langle: T_{ \pm \pm}\left(\xi_{X}^{\alpha}(X)\right):\right\rangle
$$

These two conditions are strong enough to provide a generic expression for the expectation values of the stress-energy tensor. In particular, the breaking of the classical Weyl symmetry (meaning that classically $g^{a b} T_{a b}=$ 0 ), produces a non-vanishing trace anomaly which can be derived from the above conditions. One easily obtains that

$$
\langle T\rangle=\frac{\hbar}{24 \pi}\left(R-6(\nabla \phi)^{2}+6 \square \phi\right) .
$$


The full expression for the expectation values of the stress-energy tensor components, in an arbitrary conformal coordinate system, is

$$
\begin{aligned}
\left\langle\Psi\left|T_{ \pm \pm}\left(x^{ \pm}\right)\right| \Psi\right\rangle & =-\frac{\hbar}{12 \pi}\left(\partial_{ \pm} \rho \partial_{ \pm} \rho-\partial_{ \pm}^{2} \rho\right)+\frac{\hbar}{2 \pi}\left(\partial_{ \pm} \rho \partial_{ \pm} \phi+\rho\left(\partial_{ \pm} \phi\right)^{2}\right) \\
& +\left\langle\Psi\left|: T_{ \pm \pm}\left(x^{ \pm}\right):\right| \Psi\right\rangle \\
\left\langle\Psi\left|T_{+-}\left(x^{ \pm}\right)\right| \Psi\right\rangle & =-\frac{\hbar}{12 \pi}\left(\partial_{+} \partial_{-} \rho+3 \partial_{+} \phi \partial_{-} \phi-3 \partial_{+} \partial_{-} \phi\right), \\
\left\langle\Psi\left|\frac{\delta S_{m}}{\delta \phi}\right| \Psi\right\rangle & =\left\langle\Psi\left|\frac{\delta S_{m}}{\delta \phi}\right| \Psi\right\rangle_{\rho=0}-\frac{\hbar}{2 \pi}\left(\partial_{+} \partial_{-} \rho+\partial_{+} \rho \partial_{-} \phi+\partial_{-} \rho \partial_{+} \phi\right. \\
& \left.+2 \rho \partial_{+} \partial_{-} \phi\right) .
\end{aligned}
$$

The dependence on the quantum state is all contained in the three functions $\left\langle\Psi\left|: T_{ \pm \pm}:\right| \Psi\right\rangle$ and $\left\langle\Psi\left|\frac{\delta S_{m}}{\delta \phi}\right| \Psi\right\rangle_{\rho=0}$. These functions are not independent and verify the following relations

$$
\partial_{\mp}\left\langle\Psi\left|: T_{ \pm \pm}:\right| \Psi\right\rangle+\partial_{ \pm} \phi\left\langle\Psi\left|\frac{\delta S_{m}}{\delta \phi}\right| \Psi\right\rangle_{\rho=0}-\frac{\hbar}{4 \pi} \partial_{ \pm}\left(\partial_{+} \phi \partial_{-} \phi-\partial_{+} \partial_{-} \phi\right)=0 .
$$

\subsection{Backreaction equations in the Boulware state}

In a dynamical scenario such as black hole evaporation it is highly nontrivial to unravel the precise form of the state-dependent functions $\langle\Psi|: T_{ \pm \pm}$: $|\Psi\rangle$ and $\left\langle\Psi\left|\frac{\delta S_{m}}{\delta \phi}\right| \Psi\right\rangle_{\rho=0}$. However, in this paper we are interested in static configurations, for which $\rho$ and $\phi$ are functions of the spatial coordinate $x=\left(x^{+}-x^{-}\right) / 2$ only, i.e. $\rho=\rho(x)$ and $\phi=\phi(x)$. This coordinate $x$ reduces, in the classical limit, to the tortoise coordinate $r^{*}$ given in (9). For the Boulware state it is natural to impose that

$$
\left\langle B\left|: T_{ \pm \pm}(t, x):\right| B\right\rangle=0 \text {. }
$$

This allows to determine, from Eqs. (25), the function $\left\langle\Psi\left|\frac{\delta S_{m}}{\delta \phi}\right| \Psi\right\rangle_{\rho=0}$

$$
\left\langle B\left|\frac{\delta S_{m}}{\delta \phi}\right| B\right\rangle_{\rho=0}=-\frac{\hbar}{16 \pi} \frac{\left(\phi_{x}^{2}-\phi_{x x}\right)_{x}}{\phi_{x}}
$$

where the index $x$ means derivative with respect to the coordinate $x$. Thus we have all the ingredients we need to write down the backreaction equations in 
the Boulware state, which describe how the Schwarzschild solution is modified due to pure vacuum polarization effects

$$
\begin{aligned}
\frac{2 c}{\sqrt{-g}} \frac{\delta S_{g}}{\delta g^{ \pm \pm}} & =\left\langle\Psi\left|T_{ \pm \pm}\right| \Psi\right\rangle, \\
\frac{2 c}{\sqrt{-g}} \frac{\delta S_{g}}{\delta g^{+-}} & =\left\langle\Psi\left|T_{+-}\left(x^{ \pm}\right)\right| \Psi\right\rangle, \\
-\frac{\delta S_{g}}{\delta \phi} & =\left\langle\Psi\left|\frac{\delta S_{m}}{\delta \phi}\right| \Psi\right\rangle,
\end{aligned}
$$

where

$$
\begin{aligned}
\frac{2}{\sqrt{-g}} \frac{\delta S_{g}}{\delta g^{ \pm \pm}} & =\frac{c^{3} r_{0}^{2} e^{-2 \phi}}{G}\left(\partial_{ \pm}^{2} \phi-2 \partial_{ \pm} \rho \partial_{ \pm} \phi-\left(\partial_{ \pm} \phi\right)^{2}\right) \\
\frac{2}{\sqrt{-g}} \frac{\delta S_{g}}{\delta g^{+-}} & =\frac{c^{3} r_{0}^{2} e^{-2 \phi}}{G}\left(-\partial_{+} \partial_{-} \phi+2 \partial_{+} \phi \partial_{-} \phi+\frac{1}{4 r_{0}^{2}} e^{2(\rho+\phi)}\right) \\
-\frac{\delta S_{g}}{\delta \phi} & =2 \frac{c^{3} r_{0}^{2} e^{-2 \phi}}{G}\left(\partial_{+} \partial_{-} \rho+\partial_{+} \phi \partial_{-} \phi-\partial_{+} \partial_{-} \phi\right)
\end{aligned}
$$

It is convenient to fix the constant scale $r_{0}$ as follows: $r_{0} \equiv \sqrt{\lambda}=\sqrt{\frac{l_{\text {Planck }}^{2}}{12 \pi}}=$ $\sqrt{\frac{\hbar G}{12 \pi c^{3}}}$.

The static differential equations corresponding to Eqs. (28) can then be written as

$$
\begin{aligned}
\phi_{x x}-\phi_{x}^{2}-2 \rho_{x} \phi_{x} & =e^{2 \phi}\left[\rho_{x x}-\rho_{x}^{2}+6 \rho_{x} \phi_{x}+6 \rho \phi_{x}^{2}\right] \\
\phi_{x x}-2 \phi_{x}^{2}+\frac{e^{2(\phi+\rho)}}{\lambda} & =e^{2 \phi}\left[\rho_{x x}-3\left(\phi_{x x}-\phi_{x}^{2}\right)\right] \\
\phi_{x x}-\phi_{x}^{2}-\rho_{x x} & =e^{2 \phi}\left[3 \rho_{x x}+6 \rho_{x} \phi_{x}+6 \rho \phi_{x x}+\frac{3}{2} \frac{\left(\phi_{x x}-\phi_{x}^{2}\right)_{x}}{\phi_{x}}\right] .
\end{aligned}
$$

To solve these equations we have to add boundary conditions which, in the present context, are naturally given by imposing that for very large $r$ the solution approaches the classical one (9), i.e.,

$$
\begin{aligned}
\rho & =\frac{1}{2} \ln \left(1-\frac{2 G M}{c^{2} r}\right), \\
r^{*} \equiv x & =r+\frac{2 G M}{c^{2}} \ln \left(1-\frac{2 G M}{c^{2} r}\right) .
\end{aligned}
$$


We shall investigate how the relations $\rho=\rho(r)$ and $r=r(x)$ are modified by (static) quantum effects.

It is convenient to analyze first what happens in a simplified context, defined by neglecting the coupling of the scalar field with the radial function $r$ in the classical matter action (12).

\section{Polyakov theory's approximation}

In this section we shall study a simplified version of the problem outlined in the previous section. As already mentioned we shall replace the action (12) by a new one obtained by fixing the radial function $r=r_{S}=$ constant. We then obtain

$$
S_{\text {matter }}=-\frac{1}{2} \int d^{2} x \sqrt{-g}\left|\nabla\left(r_{S} f\right)\right|^{2} .
$$

This approximation is usually motivated by arguing that, in the vicinity of the classical horizon $r \sim r_{S}=2 G M / c^{2}$, the wave equation for the scalar field

$$
\left(-\frac{\partial^{2}}{\partial t^{2}}+\frac{\partial^{2}}{\partial r^{* 2}}-V(r)\right)(r f)=0
$$

where $V(r)$ is the $s$-wave potential

$$
V(r)=\left(1-\frac{r_{S}}{r}\right) \frac{r_{S}}{r^{3}},
$$

reduces to the two-dimensional free wave equation

$$
\left(-\frac{\partial^{2}}{\partial t^{2}}+\frac{\partial^{2}}{\partial r^{* 2}}\right)\left(r_{S} f\right)=0
$$

This latter equation can indeed be derived by varying the action (35).

The expression for $\left\langle\Psi\left|T_{a b}\right| \Psi\right\rangle$ can be derived in a number of different ways. Following the arguments of subsection 2.1 one arrives at

$$
\begin{aligned}
\left\langle\Psi\left|T_{ \pm \pm}\right| \Psi\right\rangle & =-\frac{\hbar}{12 \pi}\left(\left(\partial_{ \pm} \rho\right)^{2}-\partial_{ \pm}^{2} \rho\right)+\left\langle\Psi\left|: T_{ \pm \pm}:\right| \Psi\right\rangle \\
\left\langle\Psi\left|T_{+-}\right| \Psi\right\rangle & =-\frac{\hbar}{12 \pi} \partial_{+} \partial_{-} \rho
\end{aligned}
$$

which are obtained from (22), (23) by neglecting the terms depending on

$\phi$ (note that in this approximation $\left\langle\Psi\left|\frac{\delta S_{m}}{\delta \phi}\right| \Psi\right\rangle=0$ ). Note that the above 
expressions can be also obtained from the effective Polyakov action

$$
S_{P}=-\frac{\hbar}{96 \pi} \int d^{2} x \sqrt{-g} R \square^{-1} R
$$

The dependence on the quantum state is contained in the functions $\langle\Psi|$ : $T_{ \pm \pm}\left(x^{ \pm}\right):|\Psi\rangle$, which are taken to be zero in the Boulware vacuum, i.e.

$$
\left\langle B\left|: T_{ \pm \pm}\left(x^{ \pm}\right):\right| B\right\rangle=0
$$

In the Schwarzschild background ((133) and (34) $) x^{ \pm}$denote the EddingtonFinkelstein coordinates and the components of the quantum stress tensor read

$$
\begin{aligned}
\left\langle B\left|T_{ \pm \pm}\right| B\right\rangle & =\frac{\hbar}{24 \pi}\left[-\frac{r_{S}}{2 r^{3}}+\frac{3}{8} \frac{r_{S}^{2}}{r^{4}}\right] \\
\left\langle B\left|T_{+-}\right| B\right\rangle & =-\frac{\hbar}{24 \pi}\left[1-\frac{r_{S}}{r}\right] \frac{r_{S}}{2 r^{3}} .
\end{aligned}
$$

At infinity $\left\langle B\left|T_{a b}\right| B\right\rangle \rightarrow 0$ (where $|B\rangle$ reduces to the Minkowski ground state $|M\rangle)$, while on the horizon $\left\langle B\left|T_{ \pm \pm}\right| B\right\rangle \rightarrow-\hbar c^{4} / 768 \pi M^{2} G^{2}$. These quantities are strongly divergent when expressed in Kruskal coordinates $U \sim$ $e^{-x^{-} / 2 r_{S}}, V \sim e^{x^{+} / 2 r_{S}}$ regular on the future and past horizons $\left(H^{+}\right.$and $H^{-}$, respectively)

$$
\left\langle B\left|T_{U U}\right| B\right\rangle \sim_{H^{+}} \frac{\left\langle B\left|T_{--}\right| B\right\rangle}{\left(r-r_{S}\right)^{2}}, \quad\left\langle B\left|T_{V V}\right| B\right\rangle \sim_{H^{-}} \frac{\left\langle B\left|T_{++}\right| B\right\rangle}{\left(r-r_{S}\right)^{2}} .
$$

This, in turn, means that quantum backreaction effects are strong at the classical horizon $r=r_{S}$.

The semiclassical equations in the Boulware vacuum can then be written as follows

$$
\begin{aligned}
\frac{c^{3} r_{0}^{2} e^{-2 \phi}}{G}\left(\partial_{ \pm}^{2} \phi-2 \partial_{ \pm} \rho \partial_{ \pm} \phi-\left(\partial_{ \pm} \phi\right)^{2}\right) & =-\frac{\hbar}{12 \pi}\left(\left(\partial_{ \pm} \rho\right)^{2}-\partial_{ \pm}^{2} \rho\right) \\
\frac{c^{3} r_{0}^{2} e^{-2 \phi}}{G}\left(-\partial_{+} \partial_{-} \phi+2 \partial_{+} \phi \partial_{-} \phi+\frac{1}{4 r_{0}^{2}} e^{2(\rho+\phi)}\right) & =-\frac{\hbar}{12 \pi} \partial_{+} \partial_{-} \rho \\
2 \frac{c^{3} r_{0}^{2} e^{-2 \phi}}{G}\left(\partial_{+} \partial_{-} \rho+\partial_{+} \phi \partial_{-} \phi-\partial_{+} \partial_{-} \phi\right) & =0
\end{aligned}
$$


and repeating the steps that led to Eqs. (301), (31) and (32), we rewrite them as

$$
\begin{aligned}
\phi_{x x}-\phi_{x}^{2}-2 \rho_{x} \phi_{x} & =e^{2 \phi}\left(\rho_{x x}-\rho_{x}^{2}\right), \\
\phi_{x x}-2 \phi_{x}^{2}+\frac{e^{2(\phi+\rho)}}{\lambda} & =e^{2 \phi} \rho_{x x} \\
\phi_{x x}-\phi_{x}^{2}-\rho_{x x} & =0 .
\end{aligned}
$$

We note that when the right hand side of the above equations vanishes, while keeping finite the quotient $e^{2 \phi} / \lambda \equiv r^{-2}$, we recover the classical equations and therefore the Schwarzschild solution. Due to the divergent behavior in (44), when $r$ approaches the classical horizon $r_{S}$ we expect non-trivial corrections to the classical metric.

\subsection{Decoupling the semiclassical equations}

We shall exploit the fact that Eqs. (48), (49) and (501) do not have terms that depend explicitly on the variable $x$ and, also, that (48) and (50) are homogeneous differential equations of order two. This allows to write a decoupled equation for the function $\rho(\phi)$. We use the relations

$$
\begin{aligned}
\rho_{x} & =\dot{\rho} \phi_{x}, \\
\rho_{x x} & =\ddot{\rho}\left(\phi_{x}\right)^{2}+\dot{\rho} \phi_{x x},
\end{aligned}
$$

where the dot indicates derivative with respect to $\phi$. Equation (48) can be rewritten as

$$
\phi_{x x}=\frac{1+2 \dot{\rho}+e^{2 \phi}\left[\ddot{\rho}-\dot{\rho}^{2}\right]}{1-e^{2 \phi} \dot{\rho}} \phi_{x}^{2} .
$$

Moreover, subtracting Eq.(50) from (48) we get

$$
\ddot{\rho}+\dot{\rho} \frac{\phi_{x x}}{\phi_{x}^{2}}=\frac{\dot{\rho}\left(2-e^{2 \phi} \dot{\rho}\right)}{1-e^{2 \phi}} .
$$

Equations (153) and (154) allow to obtain the desired equation

$$
\ddot{\rho}=\frac{\dot{\rho}\left[1-2 \dot{\rho}+e^{2 \phi}\left(1-\dot{\rho}+\dot{\rho}^{2}\right)\right]}{1-e^{2 \phi}}
$$

or, going back to the radial coordinate $r=r_{0} e^{-\phi}$,

$$
\rho_{r r}=-\frac{\left(1+r \rho_{r}\right) \rho_{r}\left(2 r+\lambda \rho_{r}\right)}{r^{2}-\lambda} .
$$


The equation for $\phi(x)$ can be derived by combining Eqs. (49) and (53)

$$
\phi_{x}^{2}=\frac{e^{2(\phi+\rho)}}{\lambda\left(1-2 \dot{\rho}+e^{2 \phi} \dot{\rho}^{2}\right)},
$$

and, equivalently, for $r(x)$

$$
\left(\frac{d r}{d x}\right)^{2}=\frac{e^{2 \rho}}{1+2 r \rho_{r}+\lambda \rho_{r}^{2}} .
$$

In the classical limit $(\lambda=0)$ Eqs. (56) and (58) become

$$
\rho_{r r}=-\frac{2\left(1+r \rho_{r}\right) \rho_{r}}{r}
$$

and

$$
\left(\frac{d r}{d x}\right)^{2}=\frac{e^{2 \rho}}{1+2 r \rho_{r}} .
$$

The general solution to Eq. (59) is

$$
\rho_{c}=\frac{1}{2} \ln \left(A+\frac{B}{r}\right)
$$

where $A$ and $B$ are two integration constants. The Schwarzschild metric can be easily recovered by setting $A=1$ (i.e., the metric is asymptotically Minkowskian, $\rho \rightarrow 0$ as $r \rightarrow+\infty)$ and $B=-2 G M / c^{2}$. Integration of (60) leads to the identification of $x$ with the tortoise coordinate $r^{*}$.

A natural thing would be to try to solve Eq. (56) perturbatively in $\lambda$. This gives a good approximation to the full solution when the quantum terms are small compared to the classical ones. At $O(\lambda)$ this is true for large $r$ where

$$
\lambda \rho_{r} \sim \lambda \frac{d \rho_{c}}{d r}=\frac{\lambda G M / c^{2} r^{2}}{1-2 G M / c^{2} r} \ll 2 r,
$$

but not in the near-horizon region where the quantum terms instead dominate. In this region backreaction effects are strong and cannot be treated perturbatively. 


\subsection{Numerical solution}

The differential equation (56) cannot be solved analytically. It must be studied numerically and for this it is convenient to rescale the radial coordinate and introduce the dimensionless parameter $z \equiv r / \sqrt{\lambda}$. We then get

$$
\rho_{z z}=-\frac{\left(1+z \rho_{z}\right) \rho_{z}\left(2 z+\rho_{z}\right)}{z^{2}-1} .
$$

This equation allows to analyze in a non-perturbative way the exact function $\rho(z)$. However, for reasons that will be clear in a moment, the function $\rho=\rho(z)$ is not single-valued. Therefore we have to study, instead, the function $z=z(\rho)$ which verifies the differential equation

$$
z_{\rho \rho}=\frac{\left(z+z_{\rho}\right)\left(1+2 z z_{\rho}\right)}{z^{2}-1} .
$$

Imposing as boundary condition that the solution behaves, for very large $z$, as the classical one

$$
\rho(z \rightarrow \infty)=\frac{1}{2} \ln \left(1-\frac{2 a}{z}\right)
$$

with $a \equiv G M / c^{2} \sqrt{\lambda}$, we can generate numerically the solution to (64). We find that the quantum corrected solution is everywhere similar to the classical one, up to the vicinity of the classical horizon. We can observe this behavior in Fig.1. We have chosen a black hole of small-size $\left(a=10^{3}\right)$ since in this case the differences between the classical and the semiclassical solutions can be better appreciated.

We observe that for regions far away from the classical horizon $(z>>1$, i.e. $\rho \rightarrow 0)$ the numerical solution and the classical one are very similar. However, in the vicinity of the classical horizon the quantum corrected solution suffers a bounce, absent in the classical solution, around $\rho \sim-8.3$ and then grows up slowly. This is the reason why we have had to solve numerically $z=z(\rho)$ instead of $\rho=\rho(z)$. Note that this point appears at a finite value of $\rho$, and, therefore, that the time-time component of the metric $g_{t t}$ does not vanish. The existence of this bouncing point is better represented in Fig.2, where we plot the derivative $z_{\rho}$ in terms of $\rho$. The existence of a zero for $z_{\rho}$ signals a bouncing point for the radial function.

This qualitative behavior of the radial function is maintained irrespective of the size of the black hole. For a Solar-mass black hole $\left(a=10^{39}\right)$ the bounce 


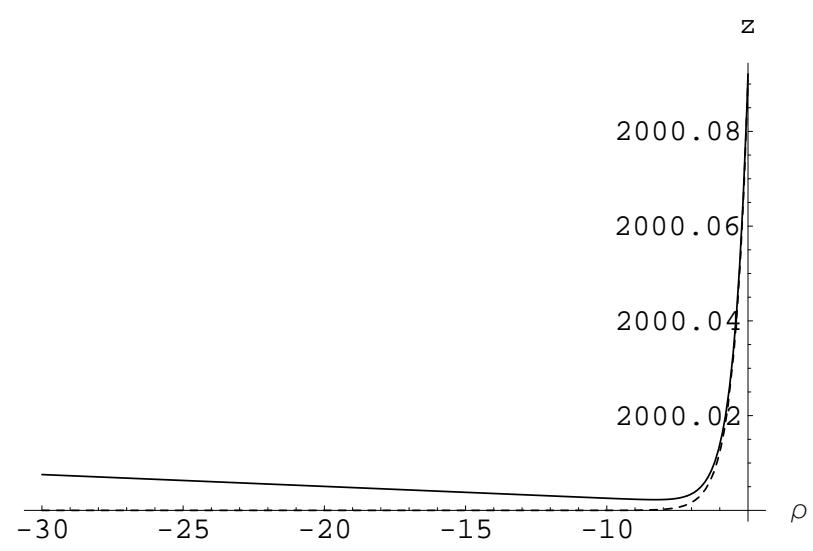

Figure 1: Classical (dashed line) and numerical (solid line) plots of the function $z(\rho)$ for $a=10^{3}$.

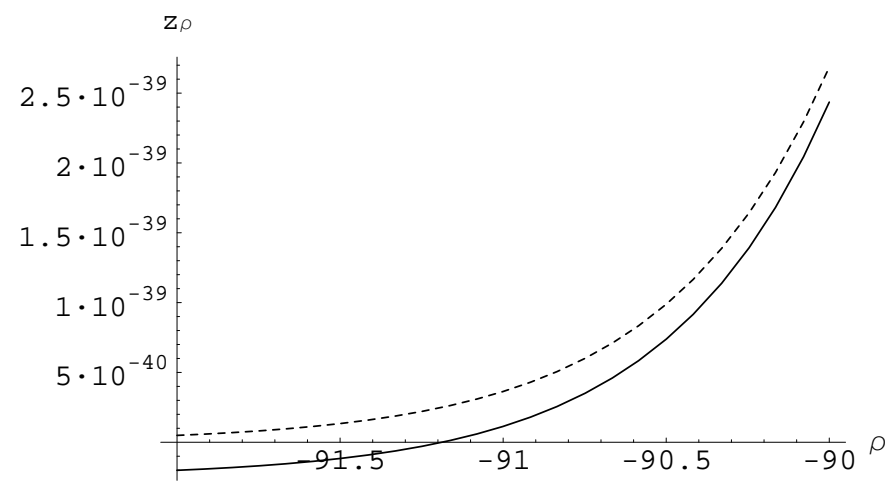

Figure 2: Plots of $z_{\rho}(\rho)$, classical (dashed) and numerical (solid) for $a=10^{39}$. 
appears at $\rho\left(z_{B}\right) \sim-91$. This result implies, as we will see later with more detail, that the classical horizon is eliminated by the quantum corrections. However, it is important to remark that the value of the conformal factor of the metric at the bouncing surface is very small. In fact the redshift for a signal emitted by a static observer at the bounce and received at infinity

$$
\frac{E_{\infty}}{E_{\text {Bounce }}}=e^{\rho\left(z_{B}\right)}
$$

transforms, for instance, a Planckian energy $E_{\text {Bounce }} \sim 10^{19} \mathrm{GeV}$ into an energy of the order $E_{\infty} \sim 10^{-12} \mathrm{eV}$ at infinity. Moreover we find that, for $a=10^{39}, z_{B}=z_{S}+8.48 \times 10^{-4}$, where $z_{S} \equiv 2 a=2 \times 10^{39}$. This shows that the bouncing surface is indeed very close to the classical horizon (the difference between $z_{B}$ and $z_{S}$ increases as one reduces the mass).

\subsection{Behavior of the metric around the bounce}

Around the bounce $z_{B}$ the function $z(\rho)$ behaves

$$
z(\rho) \approx z_{B}+\frac{1}{2} A\left(\rho-\rho_{B}\right)^{2}+\ldots .
$$

Plugging this expansion into (64) we get immediately that

$$
A=\frac{z_{B}}{z_{B}^{2}-1} .
$$

Therefore

$$
\rho(z) \approx \rho\left(z_{B}\right) \pm \sqrt{\frac{2\left(z_{B}^{2}-1\right)\left(z-z_{B}\right)}{z_{B}}},
$$

and

$$
\rho_{z} \approx \pm \sqrt{\frac{z_{B}^{2}-1}{2 z_{B}\left(z-z_{B}\right)}} .
$$

To estimate the form of the metric at $r \sim r_{B}$

$$
d s_{(4)}^{2} \approx-e^{2 \rho} c^{2} d t^{2}+e^{2 \rho}\left(\frac{d x}{d r}\right)^{2} d r^{2}+r^{2} d \Omega^{2}
$$

we need to find the relation between $r$ and $x$. From Eq. (58) we have that

$$
e^{2 \rho}\left(\frac{d x}{d r}\right)^{2}=1+2 z \rho_{z}+\rho_{z}^{2}=1+2 r \rho_{r}+\lambda \rho_{r}^{2} .
$$


In the region $r \sim r_{B}$ the right hand side is dominated by the pure quantum term $\rho_{z}^{2}=\lambda \rho_{r}^{2}$, with $\rho_{z}$ given in (70), and so we have

$$
r \approx r_{B}+\frac{1}{2} \frac{r_{B}}{r_{B}^{2}-\lambda} e^{2 \rho\left(z_{B}\right)}\left(x-x_{B}\right)^{2}+\ldots
$$

Therefore the form of the metric is approximated by

$$
d s_{(4)}^{2} \approx-e^{2 \rho} c^{2} d t^{2}+\frac{r_{B}^{2}-\lambda}{2 r_{B}^{2}} \frac{d r^{2}}{\left(1-\frac{r_{B}}{r}\right)}+r^{2} d \Omega^{2} .
$$

The quantum corrected geometry is not singular at the bounce, as it can be checked that all curvature invariants are regular at $r=r_{B}$. Note that $r$ is not the good spatial coordinate to extend the metric (174) beyond $r_{B}$, one should rather use $x$ (see (73)).

Finally we should briefly note that the surface $r=r_{B}$ is not an event horizon since $g_{t t}\left(r_{B}\right) \neq 0$. However, there $g_{r r}^{-1}\left(r_{B}\right)=0$ and this means that $\partial_{+} r^{2}<0$ for points $x$ inside $r_{B}$ and only for $r=r_{B}$ we have $\left.\partial_{+} r^{2}\right|_{r_{B}}=0$. This means that the surface $r=r_{B}$ still plays the role of an apparent horizon for outgoing radiation.

\subsection{The geometry beyond the bounce}

We shall now investigate the geometry beyond the bouncing surface. To this end we study the $2 D$ curvature for $\rho<\rho\left(z_{B}\right)$. Starting from the expression of the $2 D$ curvature

$$
R=8 e^{-2 \rho} \partial_{+} \partial_{-} \rho=-2 e^{-2 \rho} \rho_{x x},
$$

where $\rho_{x x}$ is given by (see Eq. (52) where here and in the next formulas $\dot{\rho}$ and $\ddot{\rho}$ are written in terms of derivatives with respect to $z$ )

$$
\rho_{x x}=\left(z \rho_{z}+z^{2} \rho_{z z}\right) \phi_{x}^{2}-z \rho_{z} \phi_{x x}
$$

and from (53) we get

$$
R=-2 z^{2} \frac{z \rho_{z z}+\left(2 z+\rho_{z}\right) \rho_{z}^{2}}{z+\rho_{z}} e^{-2 \rho} \phi_{x}^{2} .
$$


We can get a simplified expression for the curvature taking into account our basic differential equation (63) for $\rho(z)$

$$
\begin{aligned}
R & =-2 z^{2} \frac{\rho_{z z}}{1+z \rho_{z}} e^{-2 \rho} \phi_{x}^{2} \\
& =2 z^{2} \frac{\rho_{z}\left(2 z+\rho_{z}\right)}{z^{2}-1} e^{-2 \rho} \phi_{x}^{2} .
\end{aligned}
$$

Moreover from (58) we have

$$
e^{-2 \rho} \phi_{x}^{2}=\frac{1}{\lambda z^{2}\left(1+2 z \rho_{z}+\rho_{z}^{2}\right)}=\frac{z_{\rho}^{2}}{\lambda z^{2}\left(1+2 z z_{\rho}+z_{\rho}^{2}\right)},
$$

and therefore the final expression for the curvature is

$$
R=\frac{2}{\lambda\left(z^{2}-1\right)} \frac{\rho_{z}\left(2 z+\rho_{z}\right)}{\left(1+2 z \rho_{z}+\rho_{z}^{2}\right)}=\frac{2}{\lambda\left(z^{2}-1\right)} \frac{\left(2 z z_{\rho}+1\right)}{\left(1+2 z z_{\rho}+z_{\rho}^{2}\right)} .
$$

The natural singularity at $z=1(r=\sqrt{\lambda})$, which mimics the classical singularity at $r=0$, does not belong to the physical spacetime because of the existence of the bounce $r_{B}$ encountered before. Singularities can only be generated by zeroes of the denominator in (80). Before $r_{B}$ we have $z_{\rho}>0$, so $1+2 z z_{\rho}+z_{\rho}^{2}$ can never be zero. At $z=z_{B}$ we have $z_{\rho}=0$, so

$$
R=\frac{2}{\lambda\left(z_{B}^{2}-1\right)}
$$

After the bounce $z_{\rho}<0$ and, therefore, one can potentially encounter a curvature singularity. The numerical analysis indicates that such a singularity can be found only when $z \rightarrow+\infty$. For this we need that $\left(1+2 z z_{\rho}+z_{\rho}^{2}\right) \sim 0$ when $z \rightarrow+\infty$. The zeroes of the above second order polynomial $z_{\rho}=-z \pm$ $\sqrt{z^{2}-1}$ are an exact solution to the differential equation (64). Since the numerical analysis shows that, for large $z$ in the interior region, $z_{\rho} \sim-1 / 2 z+\ldots$ this means that asymptotically we have $z_{\rho} \sim-z+\sqrt{z^{2}-1}+f(z)$, where $z^{n} f(z) \rightarrow 0$ as $z \rightarrow+\infty$ for every positive integer number $n .{ }^{\dagger \dagger}$ The behavior of the curvature is then

$$
R \sim-\frac{1}{z^{5} f(z)}
$$

\footnotetext{
${ }^{\dagger \dagger}$ We note that the solution $z_{\rho}=-z+\sqrt{z^{2}-1}$ corresponds to the one found in 20. as an exact solution to Eqs. (48), (49) and (50) where the exponential term $e^{2(\phi+\rho)} / \lambda$ is neglected.
} 
which clearly shows the existence of a singularity at $z=+\infty$. A detailed computation, using (153), shows that (for $z \rightarrow+\infty$ )

$$
z \sim \sqrt{-x}
$$

Using Eq. (72) it is easy to realize that in the limit $z \rightarrow+\infty$ we have $f(z) \sim e^{-2 z^{2}}$ and this implies that the scalar curvature goes to $-\infty$ there as

$$
R \sim-\frac{e^{2 z^{2}}}{z^{5}}
$$

Moreover, such a singularity is null (i.e. $e^{2 \rho} \rightarrow 0$ as $z \rightarrow+\infty$ ) and is located at a finite affine distance from any finite $z$. Finally we remark that the singularity arises due to the the branching point for the radial function $r \equiv z \sqrt{\lambda}$, with respect to the spatial coordinate $x$, displayed in (83). This is the underlying reason for the generation of the curvature singularity at $x=-\infty$.

\section{Quantum corrections in the $s$ wave approx- imation.}

The approximation used in the previous section consists, essentially, in neglecting the effects of the potential barrier for the wave equation (36). In this way we have simplified considerably the technical problem. It is natural at this point to ask whether the results obtained are maintained when the effects of the potential are included. The detailed analysis presented before has allowed to introduce all the techniques that we shall use to attack the full problem in the s-wave approximation. The equations to solve are now more involved. However, since the conceptual line to follow should be clear we will focus only on the most important points.

In the Schwarzschild spacetime the expectation values of the stress tensor components in the Boulware state (see (22)-(26) and (27)) are

$$
\begin{aligned}
\left\langle B\left|T_{ \pm \pm}\right| B\right\rangle & =\frac{\hbar}{24 \pi}\left[-\frac{2 r_{S}}{r^{3}}+\frac{15}{8} \frac{r_{S}^{2}}{r^{4}}\right]+\frac{\hbar}{16 \pi r^{2}}\left(1-\frac{r_{S}}{r}\right)^{2} \ln \left(1-\frac{r_{S}}{r}\right), \\
\left\langle B\left|T_{+-}\right| B\right\rangle & =\frac{\hbar}{12 \pi}\left[1-\frac{r_{S}}{r}\right] \frac{r_{S}}{2 r^{3}}, \\
\left\langle\Psi\left|\frac{\delta S_{m}}{\delta \phi}\right| \Psi\right\rangle & =-\frac{7 \hbar}{16 \pi} \frac{r_{S}}{r^{3}}+\frac{\hbar}{2 \pi} \frac{r_{S}^{2}}{r^{4}}+\frac{\hbar}{8 \pi r^{2}}\left(1-\frac{2 r_{S}}{r}\right)\left(1-\frac{r_{S}}{r}\right) \ln \left(1-\frac{r_{S}}{r}\right) .
\end{aligned}
$$


All these quantities vanish asymptotically, while on the horizon the leading divergence is the same as in the Polyakov case (44). Again, this means that to inspect the near-horizon region $r \sim r_{S}$ we need to solve exactly the backreaction equations.

\subsection{Decoupling the semiclassical equations}

We shall now proceed in parallel to Section 3 to decouple the system of differential equations (30)-(32) to generate a single equation for $\rho=\rho(r)$. Using the relations $\rho_{x}=\dot{\rho} \phi_{x}$ and $\rho_{x x}=\ddot{\rho}\left(\phi_{x}\right)^{2}+\dot{\rho} \phi_{x x}$ we transform them into

$$
\begin{aligned}
\phi_{x x}-\phi_{x}^{2}-2 \dot{\rho} \phi_{x}^{2} & =e^{2 \phi}\left[\ddot{\rho} \phi_{x}^{2}+\dot{\rho} \phi_{x x}-\dot{\rho}^{2} \phi_{x}^{2}+6 \dot{\rho} \phi_{x}^{2}+6 \rho \phi_{x}^{2}\right] \\
\phi_{x x}-2 \phi_{x}^{2}+\frac{e^{2(\phi+\rho)}}{\lambda} & =e^{2 \phi}\left[\ddot{\rho} \phi_{x}^{2}+\dot{\rho} \phi_{x x}-3\left(\phi_{x x}-\phi_{x}^{2}\right)\right] \\
\phi_{x x}-\phi_{x}^{2}-\ddot{\rho} \phi_{x}^{2}-\dot{\rho} \phi_{x x} & =e^{2 \phi}\left[3 \ddot{\rho} \phi_{x}^{2}+3 \dot{\rho} \phi_{x x}+6 \dot{\rho} \phi_{x}^{2}+6 \rho \phi_{x x}\right. \\
& \left.+\frac{3}{2} \frac{\left(\phi_{x x}-\phi_{x}^{2}\right)_{x}}{\phi_{x}}\right] .
\end{aligned}
$$

From (86) and (87) we get

$$
\begin{aligned}
\phi_{x x}\left(1-\dot{\rho} e^{2 \phi}\right) & =\left[1+2 \dot{\rho}+e^{2 \phi}\left(\ddot{\rho}-\dot{\rho}^{2}+6 \dot{\rho}+6 \rho\right)\right] \phi_{x}^{2} \\
\phi_{x x}\left(1-(\dot{\rho}-3) e^{2 \phi}\right) & =\left[2+(\ddot{\rho}+3) e^{2 \phi}\right] \phi_{x}^{2}-\frac{e^{2(\phi+\rho)}}{\lambda}
\end{aligned}
$$

which can be rewritten as

$$
\begin{aligned}
\phi_{x}^{2} & =\frac{\left(1-\dot{\rho} e^{2 \phi}\right) e^{2(\phi+\rho)}}{\lambda D}, \\
\phi_{x x} & =\frac{\left[1+2 \dot{\rho}+e^{2 \phi}\left(\ddot{\rho}-\dot{\rho}^{2}+6 \dot{\rho}+6 \rho\right)\right] e^{2(\phi+\rho)}}{\lambda D},
\end{aligned}
$$

where

$$
\begin{aligned}
D= & -\left[1-(\dot{\rho}-3) e^{2 \phi}\right]\left[1+2 \dot{\rho}+e^{2 \phi}\left(\ddot{\rho}-\dot{\rho}^{2}+6 \dot{\rho}+6 \rho\right)\right] \\
& +\left[1-\dot{\rho} e^{2 \phi}\right]\left[2+(\ddot{\rho}+3) e^{2 \phi}\right] .
\end{aligned}
$$

Taking into account that

$$
\frac{\phi_{x x x}}{\phi_{x}}=\dot{\phi}_{x x}
$$


and plugging the expressions (91) and (92) into (88) we obtain, after a straightforward but very long calculation, a third-order differential equation relating $\rho$ and $r$

$$
\begin{aligned}
& {\left[r^{2}+3 \lambda-6 \lambda \rho+r \rho_{r}\left(2 r^{2}+6 \lambda+r \lambda \rho_{r}\right)\right] \times\left[72 \lambda^{2} \rho^{2}\left(r^{2}+3 \lambda+r \lambda \rho_{r}\right)+\right.} \\
& +6 r \lambda \rho\left(12 r \lambda-\rho_{r}\left(6 r^{4}+40 r^{2} \lambda+63 \lambda^{2}+2 r \lambda \rho_{r}\left(4 r^{2}+14 \lambda+r \lambda \rho_{r}\right)\right)+\right. \\
& \left.+r \lambda\left(2 r^{2}+15 \lambda+2 r \lambda \rho_{r}\right) \rho_{r r}\right)+r^{2}\left(r \lambda\left(6 r^{4}+56 r^{2} \lambda+93 \lambda^{2}\right) \rho_{r}^{3}+\right. \\
& +2 r^{2} \lambda^{2}\left(r^{2}+6 \lambda\right) \rho_{r}^{4}+\rho_{r}^{2}\left(4 r^{6}+54 r^{4} \lambda+162 r^{2} \lambda^{2}+162 \lambda^{3}-9 r^{2} \lambda^{3} \rho_{r r}\right)+ \\
& +r^{2}\left(2\left(r^{4}+5 r^{2} \lambda+12 \lambda^{2}\right) \rho_{r r}+9 \lambda^{3} \rho_{r r}^{2}-3 r \lambda^{2} \rho_{r r r}\right)+r \rho_{r}\left(4 r^{4}-6 r^{2} \lambda-\right. \\
& \left.\left.\left.72 \lambda^{2}+\lambda\left(2 r^{4}-14 r^{2} \lambda-69 \lambda^{2}\right) \rho_{r r}-3 r \lambda^{3} \rho_{r r r}\right)\right)\right]=0
\end{aligned}
$$

The second factor in the above equation is the relevant one since it leads directly, when $\lambda=0$, to the classical equation (59). The quantum corrections to the Schwarzschild metric should be then computed by exactly solving the above differential equation. Introducing the dimensionless coordinate $z=e^{-\phi}=r / \sqrt{\lambda}$ we get an ordinary differential equation for $\rho=\rho(z)$. However, as we have already explained in the analysis of the Polyakov theory, since the function $\rho=\rho(z)$ could not be one-to-one it is more appropriate to work directly with the differential equation for the function $z=z(\rho)$. It reads as follows

$$
\begin{aligned}
& 18 \rho(-21+4 \rho) z z_{\rho}^{4}+216 \rho^{2} z_{\rho}^{5}+2 z^{8} z_{\rho}^{2}\left(2 z_{\rho}-z_{\rho \rho}\right)+2 z^{7} z_{\rho}\left(3 z_{\rho}+2 z_{\rho}^{3}\right. \\
& \left.-z_{\rho \rho}\right)+3 z^{3} z_{\rho}\left((31-4 \rho) z_{\rho}-8(3+10 \rho) z_{\rho}^{3}+(23-4 \rho) z_{\rho \rho}\right) \\
& +6 z^{2} z_{\rho}^{2}\left((27-28 \rho) z_{\rho}+12 \rho(1+\rho) z_{\rho}^{3}-15 \rho z_{\rho \rho}\right)+2 z^{6} z_{\rho}\left(1+27 z_{\rho}^{2}\right. \\
& \left.-5 z_{\rho} z_{\rho \rho}\right)-3 z^{4}\left(-3 z_{\rho \rho}+2 z_{\rho}\left(-2+(-27+8 \rho) z_{\rho}^{2}+2(2+\rho) z_{\rho} z_{\rho \rho}\right)\right. \\
& \left.-z_{\rho \rho \rho}\right)+z^{5}\left(56 z_{\rho}^{2}-6(1+6 \rho) z_{\rho}^{4}-9 z_{\rho \rho}^{2}+z_{\rho}\left(14 z_{\rho \rho}+3 z_{\rho \rho \rho}\right)\right)=0 .
\end{aligned}
$$

\subsection{Numerical solution}

We solve numerically the above equation by imposing that, for very large $z$ and $\rho \rightarrow 0$, the solution approaches the classical one. We find that the solution is almost identical to the classical one up to the vicinity of a surface, 
which we also denote as $z_{B}$, located very close to the classical horizon. The result (for a Solar-mass black hole $a \equiv G M / c^{2} \sqrt{\lambda}=10^{39}$ ) is depicted in Fig. 3 which shows the existence of a bounce for the radial function at

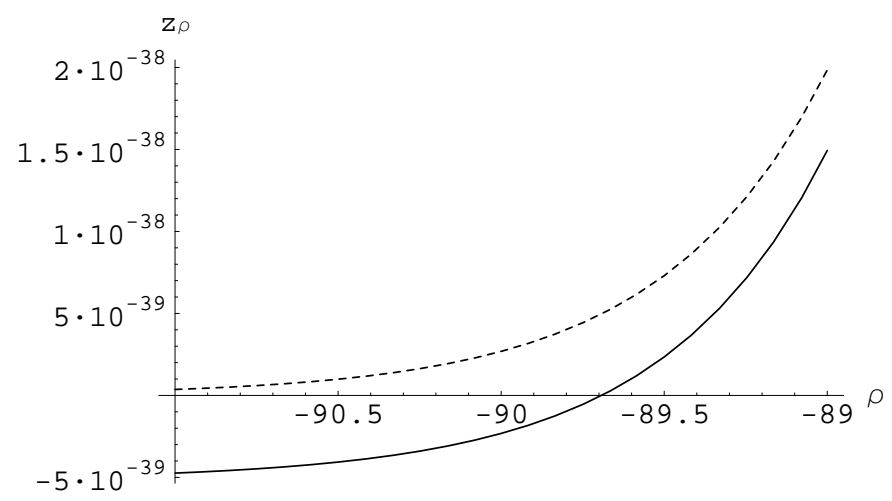

Figure 3: Plots of the function $z_{\rho}(\rho)$, classical (dashed line) and numerical (solid line) for $a=10^{39}$.

$\rho_{B} \approx-89.69$, where

$$
z(\rho) \approx z_{B}+\frac{1}{2} A\left(\rho-\rho_{B}\right)^{2}+\ldots
$$

and $A$ is a positive coefficient to be computed numerically. Moreover, we find that $z_{B} \approx z_{S}+1.76 \times 10^{-37}$. Therefore, its location is much closer to the classical horizon than in the Polyakov theory approximation of section 3.

\subsection{Branching point for the radial function}

The main difference with respect to the Polyakov theory appears in the relation between $r$ and $x$. In terms of the function $z(\rho)$ we have

$$
\begin{aligned}
e^{2 \rho}\left(\frac{d x}{d r}\right)^{2} & =\frac{z z_{\rho} D}{\left(1+z z_{\rho}\right)} \\
e^{2 \rho}\left(\frac{d x}{d \rho}\right)^{2} & =\frac{\lambda z z_{\rho}^{3} D}{\left(1+z z_{\rho}\right)}
\end{aligned}
$$


where

$$
\begin{aligned}
D=\frac{1}{z^{4} z_{\rho}^{4}}\{ & -\left[z^{2} z_{\rho}+z+3 z_{\rho}\right]\left[z^{2} z_{\rho}^{3}-2 z^{3} z_{\rho}^{2}-z^{2} z_{\rho \rho}-5 z z_{\rho}^{2}-z^{2} z_{\rho}+6 \rho z_{\rho}^{3}\right] \\
& \left.+\left[z^{2} z_{\rho}+z\right]\left[2 z^{2} z_{\rho}^{3}+3 z_{\rho}^{3}-z^{2} z_{\rho \rho}+z z_{\rho}^{2}\right]\right\} .
\end{aligned}
$$

In Fig. (41) we show the typical behavior of the function $D$ in terms of $\rho$.

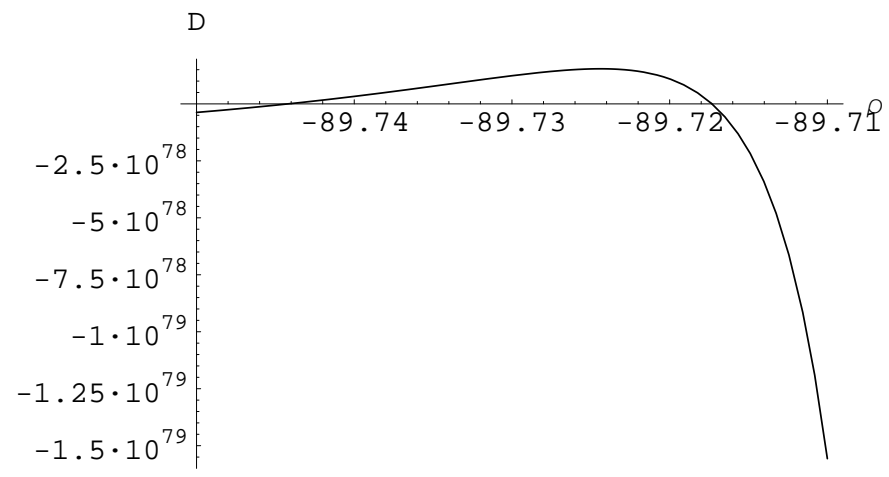

Figure 4: For $a=10^{39}$ the bounce of the radial function $(D=+\infty)$ is located at $\rho \sim-89.69$. At $\rho \sim-89.72$ the function $D$ vanishes.

Note that in the vicinity of the bounce $z_{\rho} \rightarrow 0$, and from (100) we have $D \sim z_{\rho}^{-3}$. Therefore

$$
e^{2 \rho}\left(\frac{d x}{d r}\right)^{2} \sim \frac{1}{z_{\rho}^{2}}
$$

and hence

$$
\frac{d r}{d x} \sim z_{\rho}
$$

In other words, the expansion of $r$ in terms of $x$ has to be of the form $r \approx r_{B}+\alpha\left(x-x_{B}\right)^{2}+\ldots$, where $\alpha$ is a numerical constant, in agreement with the analytic behavior encountered in (73) .

A similar argument allows to determine the behavior of $r$ in terms of $x$ around the zero of the function $D$. This happens at $\rho=\rho_{M} \lesssim \rho_{B}$, just after the bounce. Around the zero of $D$ we have $D(r) \sim r_{M}-r$. Therefore

$$
\frac{d x}{d r} \sim-\sqrt{r_{M}-r}
$$


and hence

$$
r \approx r_{M}-\beta\left(x-x_{M}\right)^{2 / 3}
$$

where $\beta$ is a numerical positive constant. The radial function has a branching point at $x=x_{M}$, which turns out to be the minimum possible value for the "tortoise" coordinate $x$.

The form of the metric in this region is

$$
d s_{(4)}^{2} \approx e^{2 \rho(r)}\left(-c^{2} d t^{2}+d x^{2}\right)+\left(r_{M}+B\left(x-x_{M}\right)^{2 / 3}\right)^{2} d \Omega^{2}
$$

where, according to our previous analysis, the function $\rho(r)$ is finite and regular at $r_{M}$. The above metric has a singularity at $r=r_{M}$, which cannot be avoided by a change of coordinates. It is indeed a curvature singularity as we now show. The $4 D$ scalar curvature can be expressed, in terms of $\rho$ and $\phi$, as follows

$$
R^{(4)}=-2 e^{-2 \rho}\left[\rho_{x x}-2 \phi_{x x}+3 \phi_{x}^{2}\right]+2 e^{2 \phi} .
$$

The first term is just the two-dimensional scalar curvature $R=-2 e^{-2 \rho} \rho_{x x}$, which according to (76), (911) and (92) is

$$
R=-\frac{2}{\lambda z^{2} D} \frac{-z^{3} z_{\rho \rho}+2 z^{3} z_{\rho}+z^{2}+6 z z_{\rho}-6 \rho z_{\rho}}{z z_{\rho}^{3}} .
$$

At $x=x_{M}$, where $D\left(x_{M}\right)=0, R$ and also the second and third terms in (106) are divergent. Finally we note, from (105), that this singularity is timelike, has finite radius $r_{M}$ and is located at a finite affine distance away.

\section{Conclusions}

The existence of a bounce for the radial function $r$, prior to the emergence of a spacetime singularity, is perhaps the most significative result of our analysis. It already appears in the simplified Polyakov theory and it is still there in the most accurate $s$-wave approximation. It is natural to expect it to persist in a full treatment of the problem.

Our results are perhaps not surprising from the semiclassical point of view, where due to the strong divergence of the Boulware stress tensor at the Schwarzschild horizon important deviations from the classical behavior are indeed expected to arise. However they constitute an important prediction 
for the search of static 5D braneworld configurations with asymptotically flat boundary conditions on the brane: such solutions are not black holes, but rather naked singularities. In the $4 \mathrm{D}$ semiclassical context this reinforces the idea that the Boulware state describes the vacuum polarization around a static star, not a black hole. Indeed the natural thing for a black hole is to be time dependent and to evaporate via the Hawking effect.

Finally we point out that our analysis does not exclude the existence of static braneworld black holes, but the price to pay is to give up asymptotic flatness on the brane. This means, in the dual $4 D$ theory, to replace Boulware with the Hartle-Hawking state.

\section{Acknowledgements}

We thank R. Balbinot, S. Fagnocchi and G.P. Procopio for useful discussions and J.M. Martin-Garcia, A. Nagar, M. Nebot and C. Talavera for assistance in the numerical analysis.

\section{References}

[1] S. W. Hawking, Comm. Math. Phys. 43199 (1975)

[2] L. Parker Phys. Rev. D 121519 (1975)

[3] R. M. Wald Comm. Math. Phys. 459 (1975)

[4] S.W. Hawking, Phys. Rev. D14, 2460 (1976)

[5] N. D. Birrel and P. C. W. Davies Quantum fields in curved spaces, Cambridge University Press, Cambridge (1982)

[6] V. P. Frolov and I. D. Novikov Black hole physics, Kluwer Academic Publisher, Dordrecht (1996)

[7] A. Fabbri and J. Navarro-Salas Modeling black hole evaporation, Imperial College Press-World Scientific, London (2005)

[8] See for instance G.'t Hooft, Nucl. Phys. B335, 138 (1990); T. Banks, A. Dabholkar, M.R. Douglas and M. O'Loughlin, Phys. Rev. D45, 3607 (1992); K. Schoutens, H.L. Verlinde and E.P. Verlinde, Phys. Rev. D48, 2670 (1993); L. Susskind, L. Thorlacius and J. Uglum, Phys. Rev. D48, 
3743 (1993); C.R. Stephens, G. 't Hooft and B.F. Whiting, Class. Quant. Grav. 11, 621 (1994); D.A. Lowe, J. Polchinski, L. Susskind, L. Thorlacius and J. Uglum, Phys. Rev. D52, 6997 (1995); R.C. Myers, Gen. Rel. Grav. 29, 1217 (1997); D. Amati, J. Phys. G29, 31 (2003); G.T. Horowitz and J. Maldacena, JHEP 0402, 008 (2004); A. Ashtekar and M. Bojowald, Class. Quant. Grav. 22, 3349 (2005)

[9] S.W. Hawking, Information loss in black holes, hep-th/0507171

[10] D. Boulware, Phys. Rev. D11, 1404 (1975)

[11] S.M. Christensen and S.A. Fulling, Phys. Rev.D15, 2088 (1977)

[12] J.B. Hartle and S.W. Hawking, Phys. Rev.D13, 2188 (1976); W. Israel, Phys. Lett.A57, 107 (1976)

[13] W.G. Unruh, Phys. Rev.D14, 870 (1976)

[14] L. Randall and R. Sundrum, Phys. Rev. Lett. 83, 4690 (1999)

[15] O. Aharony, S. Gubser, J. Maldacena, H. Ooguri and Y. Oz Phys. Rept. 323, 183 (2000)

[16] A. Chamblin, S.W. Hawking and H.S. Reall, Phys. Rev. D61, 065007 (2000); N. Dadhich, R. Maartens, P. Papadopoulos and V. Rezania, Phys. Lett. B487, 1 (2000); M. Bruni, C. Germani and R. Maartens, Phys. Rev. Lett. 87, 231302 (2001); P. Kanti and K. Tamvakis, Phys. Rev. D65, 084010 (2002); R. Casadio, A. Fabbri and L. Mazzacurati, Phys. Rev. D65, 084040 (2002); G. Kofinas, E. Papantonopoulos and V. Zamarias, Phys. Rev. D66, 104028 (2002); H. Kudoh, T. Tanaka and T. Nakamura, Phys. Rev. D68, 024035 (2003); C. Charmousis and R. Gregory, Class. Quant. Grav. 21, 527 (2004)

[17] R. Emparan, A. Fabbri and N. Kaloper, JHEP 08, 043 (2002); T. Tanaka, Prog. Theor. Phys. Suppl. 148, 307 (2003)

[18] P.R. Anderson, R. Balbinot and A. Fabbri, Phys. Rev. Lett. 94, 061301 (2005); J. Garriga and T. Tanaka, Phys. Rev. Lett. 84, 2778 (2000); M.J. Duff and J.T. Liu, Phys. Rev. Lett. 85, 2052 (2000)

[19] P.R. Anderson, W.A. Hiscock and D.A. Samuel, Phys. Rev. D51, 4337 (1995) 
[20] D.A. Lowe, Phys. Rev. D47, 2446 (1993) 